\title{
The Prevalence of Hilar Lymphadenopathy and Related Factors in Pneumoconiosis Patients of a University Hospital Occupational Diseases Polyclinic
}

Yusuf Samir Hasanlı', [MD]

ORCID: 0000-0001-6514-6789

Meral Türk', [MD]

ORCID: 0000-0002-1288-7097
${ }^{1}$ Ege University Faculty of Medicine, Department of Internal Diseases, Department of Occupational Diseases, İzmir.

${ }^{2}$ Ege University Faculty of Medicine, Department of Public Health, Department of Occupational Diseases, İzmir.

Corresponding Author: Yusuf Samir Hasanlı Ege University Faculty of Medicine, Department of Internal Diseases, Department of Occupational Diseases, İzmir

E-mail: dryusufsmrh@gmail.com

https://doi.org/10.32552/2021.ActaMedica.595

\section{nee) ABSTRACT Cen}

Objective: Pneumoconiosis is a fatal, irreversible interstitial lung disease caused by various inorganic dust and chemicals. The disease is radiologically characterized by micronodules, pleural plaques, emphysema, mediastinal and hilar lymphadenopathies (HL). There are hypotheses that fibrotic lymph nodes increase the dust load of the lungs in patients with pneumoconiosis. Our aim is to determine the frequency of $\mathrm{HL}$ and related factors in pneumoconiosis patients admitted to our unit in the light of current data.

Material and Methods: 76 patients diagnosed with pneumoconiosis who applied to our unit between November, 2015 and October, 2020 were retrospectively screened. Sociodemographic characteristics, radiological, laboratory and clinical findings were evaluated. The frequency of $\mathrm{HL}$ and related factors were analyzed.

Results: We found the rate of $\mathrm{HL}$ as $29,3 \%$. The average age was 43,78 $\pm 7,54$. There was no significant age difference between patients with and without HL $(44,41 \pm 8,90 / 43,34 \pm 6,93 p=0,578)$. All of the patients were male. As the ILO (International Labor Organization) category rose, we saw an increase in the number of HL. $59,1 \%$ of the patients with $\mathrm{HL}$ had at least one active complaint (dyspnea, cough or sputum). The most common complaint was dyspnea (50\%). We found a statistically significant increase in NLR (Neutrophil to Lymphocyte Ratio) and TLR (Thrombocyte to Lymphocyte Ratio) values in patients with $\mathrm{HL}$ compared to those without $(2,38 \pm 0,70 ; 1,97 \pm 0,59 \mathrm{p}=0,016$ and $133,79 \pm 39,06 ; 109,03 \pm 29,00 p=0,005)$. Another important finding was that the albumin values of patients with $\mathrm{HL}$ were found to be statistically significantly lower $(4.42 \pm 0.41 ; 4.67 \pm 0.29 p=0.008)$.

Conclusion: $29.3 \%$ of patients had HL. We found significant increase in NLR and TLR in pneumoconiosis patients with HL and decrease in serum albumin levels. We think that these markers may have prognostic value in pneumoconiosis patients with lymphadenopathy.

Keywords: Pneumoconiosis, hilar lymphadenopathy, neutrophil to lymphocyte ratio, platelet to lymphocyte ratio, neutrophil, lymphocyte, albumin.

Received: 30 April 2021, Accepted: 26 August 2021,

Published online: 6 November 2021 


\section{INTRODUCTION}

Some occupational groups pose a risk for the development of lung diseases due to dust and chemicals taken by air. Pneumoconiosis, known as dust disease of the lungs, which is completely preventable, is a reaction of tissues to accumulated inorganic dust [2]. Workers in environments with insufficient ventilation and continuous and intense dust exposure are at serious risk. Currently, tissue diagnosis is not used in the diagnosis of pneumoconiosis. Diagnosis can be made with a well taken anamnesis and imaging. There are many types of pneumoconiosis; such as coal worker pneumoconiosis, silicosis, asbestosis, siderosis. Prolonged exposure to dusts such as silica leads to persistent inflammation and fibrosis; this event is irreversible and fatal $[1,16]$. The pathophysiology of silicosis is a complex inflammatory process. Respired crystallized silica leads to the activation of the phagosomes and causes them to become unstable. Inflammatory cytokines (TNF-alpha, IL-1), cytotoxic oxidants, proteases and arachidonic acid metabolites are synthesized. In addition, reactive oxygen radicals emerge. As a result of all these chain events, activated macrophages and various mesenchymal cells start to produce excessive collagen and interstitial lung fibrosis occurs [5].

Today, the standardization of the International Labor Organization (ILO) is used to evaluate radiographic abnormalities in pneumoconiosis patients. Basically, both the parenchyma and the pleural area are subjected to a certain evaluation and categorized on the chest $x$-ray [5]. ILO categories are given in Table 1.

In recent years, the use of computed tomography has increased to show the radiological abnormalities of pneumoconiosis and to distinguish it from other lung diseases. High Resolution Computed Tomography (HRCT) is more commonly used. Pneumoconiosis findings in $\mathrm{HRCT}$; centrilobular micronodules, subpleural nodule, pleural plaque, areas of emphysema, diffuse interstitial pulmonary fibrosis and lymphadenopathy (mediastinal, hilar) $[5,18]$. Computed tomography is the method of choice for the evaluation of lymphadenopathy, as it is able to demonstrate increased size of individual nodes, abnormalities of the interface between the mediastinum and lung, invasion of surrounding fat, coalescence of adjacent nodes, obliteration of the mediastinal fat, and hypo- and hyperdensity in lymph nodes [7]. Cox-Grasner et al., in their study on subjects with parenchymal silicosis, found that $90 \%$ of the cases were accompanied by fibrotic lymph nodes. They thought that fibrosis in the lymph node prevented the removal of silica dust from the lungs, increased the dust load of the lung, and posed an additional risk for parenchymal silicosis $[7,8]$.

\section{MATERIAL and METHODS}

\section{Datasource and Study Population}

Before starting the study, approval was obtained from the Ethics Committee of Ege University (decision no:21-2.1T/49 and date:18.02.2021). This study is a cross-sectional, retrospective study conducted on patients diagnosed with pneumoconiosis who applied to our department between November, 2015 and October, 2020. International Labor Organization (ILO) standardization was used in the diagnosis of pneumoconiosis. ILO categorization is the international standardization method of radiography. It was revised in 2011. It is a method of collecting pneumoconiosis patients in categories (Table 1). Patients in categories 1, 2 and 3 according to the ILO evaluation were included in the study. Category 0 patients were excluded from the study. Patients with acute infection, severe allergy and critical illness were excluded from the study as it would affect laboratory results and lymphadenopathy status. Among confounding factors, such as anemia (for dyspnea), infection (for cough, sputum) were previously excluded. Patients' age, occupation, smoking status, duration of work, complaints, radiological imaging, hemogram and biochemistry results were included in the study.

Table 1. ILO Pneumoconiosis Classification

\begin{tabular}{|c|c|c|c|c|c|c|c|c|c|c|c|c|}
\hline \multicolumn{13}{|c|}{ Increase in small opacity profusion } \\
\hline Categories & \multicolumn{3}{|c|}{0} & \multicolumn{3}{|c|}{1} & \multicolumn{3}{|c|}{2} & \multicolumn{3}{|c|}{3} \\
\hline Sub Categories & $0 /-$ & $0 / 0$ & $0 / 1$ & $1 / 0$ & $1 / 1$ & $1 / 2$ & $2 / 1$ & $2 / 2$ & $2 / 3$ & $3 / 2$ & $3 / 3$ & $3 /+$ \\
\hline
\end{tabular}




\section{Statistical Analysis}

In the analysis of data, IBM SPSS for Windows v.24.0 program was used. Categorical variables were presented in cross-tables and numerical variables in mean, median, standard deviation, minimum and maximum. In the comparison of independent categorical variables, Chi square test was used. The existence of a statistically significant difference was examined with Student's $t$ test for the parametric among the groups in terms of continuous variables, and the Mann-Whitney $U$ test for nonparametric variables. Pearson's correlation analysis was also performed. $\mathrm{P}$ value of $<0.05$ was considered statisticallly significant.

\section{RESULTS}

The number of patients included in our study consisted of 76 people. All of the patients were male. The mean age of all our patients was 43.78 \pm 7.54 years (Table 5). We found the rate of hilar lymphadenopathy to be $29.3 \% \quad(n=22)$. One patient had no Computed Tomography (HRCT) result. Chi-square test was performed for the relationship between ILO categories and hilar lymphadenopathy. There was a significant relation between hilar lymphadenopathy status and the ILO category $(p<0,001)$. Hilar lymphadenopathy was most common in ILO category 3 patients (4, 8 and 10 patients in categories 1,2 and 3 , respectively). Most of the patients without hilar lymphadenopathy were collected in ILO category 1 $(n=38)$. Hilar lymphadenopathy was more common while the ILO category increased (Table 2).

The mean age of all our patients was $43.78 \pm$ 7.54 years (Table 5). There was no significant age difference between those with / without hilar lymphadenopathy $(44.41 \pm 8.90 / 43.34 \pm 6.93$ $p=0,578$ ). The majority of the patients were found

Table 2. The relationship between hilar lymphadenopathy and ILO categories.

\begin{tabular}{|c|c|c|c|}
\hline & & \multicolumn{2}{|c|}{ Hilar Lymphadenopathy } \\
\hline & & Yes & No \\
\hline \multirow{3}{*}{ İOO Category } & Cat 1 & $4(\% 9,5)$ & $38(\% 90,5)$ \\
\hline & Cat 2 & $8(\% 34,8)$ & $15(\% 65,2)$ \\
\hline & Cat 3 & $10(\% 100)$ & $0(\% 0)$ \\
\hline \multicolumn{2}{|l|}{ Total } & $22(\% 29,3)$ & $53(\% 70,7)$ \\
\hline
\end{tabular}

Pearson's Chi square

$p<0,001^{*} \quad p<0.000^{*}$ to be ceramic industry workers $(n=37(\% 48.7))$, miners $(n=21(\% 27.6))$, and grinding workers $(n=12(\% 15.3))$. We found that $89.5 \%$ of the patients worked in workplaces suspected of causing pneumoconiosis for 10 years or more. We noted this period as the exposure time (Table 3).

$59.1 \%$ of the patients with hilar lymphadenopathy had at least one active complaint (dyspnea, cough or sputum). The most common finding was dyspnea at $50 \%$ (11 of 22 patients). $67.9 \%$ of the patients without hilar lymphadenopathy (36 of 53 patients) had no active complaints. We found a positive relationship between the hilar lymphadenopathy status and the patient being symptomatic $(p=0,03)$ (Table 4).

$80.3 \%$ of our patients have smoked for at least 5 pack/years. Smoking exposure was significantly higher in patients with hilar lymphadenopathy $(18.86 \pm 16.82$ and $11.60 \pm 8.42$ pack years, respectively, $p=0.015$ ). No significant difference was found between the exposure times of patients with and without hilar lymphadenopathy (17.00 \pm 7.74 and $18.08 \pm 7.19$ years, respectively, $p=0.566$ ). See Tables 5 and 6 .

Although the spirometry values of the patients with hilar lymphadenopathy were lower, only the decrease in the FVC\% value was statistically significant: FEV1 (respectively, liters; $3.08 \pm 0.68$ / $3.29 \pm 0.53 \mathrm{p}=0.154$ and percent; $84.5 \pm 16.5$ / $91.2 \pm 11.9 p=0.054$ ) and FVC (respectively, liters; $3.85 \pm 0.85 / 4.15 \pm 0.71 \mathrm{p}=0.121$ and percent; $87.1 \pm 17.4 / 94.4 \pm 12.0 p=0.044)$. No significant change was found in the FEV1 / FVC ratio (80.6 \pm 8.3 and $80.2 \pm 7.6 p=0.832$ ). In patients with hilar lymphadenopathy, lymphocyte count was significantly lower $(2081 \pm 545 ; 2444 \pm 599 p=$ 0.022), and neutrophil to lymphocyte ratio (NLR) was significantly higher $(2.38 \pm 0.70 ; 1.97 \pm 0.59 p=$ 0.016), the thrombocyte to lymphocyte ratio (TLR) was significantly higher (133.79 \pm 39.06; $109.03 \pm$ $29.00 \mathrm{p}=0.005$ ). No statistically significant change was found in the leukocyte, platelet and neutrophil counts. In patients with hilar lymphadenopathy, the albumin value was low $(4.42 \pm 0.41 ; 4.67 \pm$ $0.29 p=0.008)$ and the sedimentation value was significantly higher $(4.73 \pm 2.81 ; 3.23 \pm 1.69 \mathrm{p}=$ 0.024). Although C-reactive protein was higher in patients with hilar lymphadenopathy, it was not statistically significant $(0.39 \pm 0.31 ; 0.24 \pm 0.23 p=$ 0.089). See Table 6. 
Table 3. Patient distribution frequency in the workplace, exposure time and smoking status.

\begin{tabular}{|l|c|c|c|c|}
\hline & \multicolumn{4}{|c|}{$\boldsymbol{n ~ ( \% )}$} \\
\hline \multirow{2}{*}{ Workplace } & Ceramic & Miner & Grinding & Dental Laboratory \\
& $37(\% 48,7)$ & $21(\% 27,6)$ & $12(\% 15,3)$ & $6(\% 7,9)$ \\
\hline \multirow{2}{*}{ Exposure Time (year) } & $\leq \mathbf{9}$ & $\mathbf{1 0 - 1 9}$ & $\geq \mathbf{2 0}$ & \\
& $8(\% 10,5)$ & $38(\% 50)$ & $30(\% 39,5)$ & \\
Smoking (pack year) & $<\mathbf{5}$ & $\mathbf{2 5}$ & & \\
& $15(\% 19,7)$ & $61(\% 80,3)$ & & \\
\hline
\end{tabular}

n: number of patients

Table 4. Relationship between hilar lymphadenopathy and patient complaint.

\begin{tabular}{|c|c|c|c|c|}
\hline & & \multicolumn{2}{|c|}{ Active Complaint } & \multirow{2}{*}{ Total } \\
\hline & & No & Yes & \\
\hline \multirow{2}{*}{ Hilar Lymphadenopathy } & Yes & $9(\% 40,9)$ & $* 13(\% 59,1)$ & 22 \\
\hline & No & $36(\% 67,9)$ & $* * 17(\% 32,1)$ & 53 \\
\hline \multicolumn{2}{|l|}{ Total } & $45(\% 60)$ & $30(\% 40,1)$ & 75 \\
\hline \multicolumn{2}{|l|}{ Pearson's Chi square } & \multicolumn{3}{|c|}{$\mathrm{p}=0,030^{* * *}$} \\
\hline \multirow{2}{*}{\multicolumn{2}{|c|}{$p<0,05^{* * *}$}} & \multicolumn{3}{|c|}{ * 11 of 13 patients had dyspnea } \\
\hline & & \multicolumn{3}{|c|}{ **11 of 17 patients had dyspnea } \\
\hline
\end{tabular}

Table 5. General frequency of pneumoconiosis patients regarding age, exposure time and smoking status.

\begin{tabular}{|l|c|c|c|c|}
\hline & $\mathbf{n}$ & mean \pm sd & min & max \\
\hline Age (year) & 76 & $43,78 \pm 7,54$ & 22 & 69 \\
\hline Exposure Time (year) & 76 & $17,68 \pm 7,30$ & 5 & 50 \\
\hline Smoking (packyear) & 76 & $13,55 \pm 11,91$ & 0 & 60 \\
\hline
\end{tabular}

n: number of patients

sd: standart deviation

\section{DISCUSSION}

The aim of our study was to determine the frequency of hilar lymphadenopathy in patients with pneumoconiosis and the factors it may be associated with. Lymphadenopathy is a common radiological finding in many thoracic diseases and may be caused by a variety of infectious, inflammatory, and neoplastic conditions. In silicosis, hilar and mediastinal lymph node enlargement may precede the appearance of parenchymal nodular lesions. This means a separate risk situation for parenchymal involvement [19]. Murray et al. found that the prevalence of parenchymal silicosis was five times higher in those with lymph node silicosis (43.3\%) than those without lymph node silicosis (7.9\%) [13]. Calcification of lymph nodes is common and typically occurs around the node. The pattern of eggshell calcification suggests silicosis. In a retrospective cross-sectional study on pneumoconiosis consisting of 61 patients, the frequency of hilar lymphadenopathy was found to be $42.6 \%$ [2]. In another study on dental technicians, this rate was found to be $28.6 \%$ [3]. In the study on coal workers, the frequency of hilar lymphadenopathy was reported as 30\% [1]. In our study, this frequency was $29.3 \%$.

Over the past few decades, there has been increasing evidence that systemic inflammation plays an important role in the pathophysiology of pneumoconiosis. The inflammatory reaction that occurs in tissue injury is an effective strategy, and can be harmful in case of elongation. Laboratory markers such as sedimentation, C-reactive protein, NLR and TLR are used to evaluate inflammation [4]. The neutrophil to lymphocyte ratio has been recognized as a simple and reliable inflammatory marker [9]. It has been shown to be useful in the classification of mortality in major cardiac events, as a strong prognostic factor in various types of cancer (such as colorectal, breast, lung, renal cell carcinoma) or as an indicator and marker 
Table 6. The relationship between hilar lymphadenopathy and different variables.

\begin{tabular}{|c|c|c|c|c|}
\hline Variables & Hilar LAP & $\mathbf{n}$ & Mean \pm SD & p value \\
\hline \multirow{2}{*}{ Age (year) } & Yes & 22 & $44,41 \pm 8,90$ & \multirow{2}{*}{$* 0,578$} \\
\hline & No & 53 & $43,34 \pm 6,93$ & \\
\hline \multirow{2}{*}{ Exposure Time (year) } & Yes & 22 & $17,00 \pm 7,74$ & \multirow{2}{*}{$* 0,566$} \\
\hline & No & 53 & $18,08 \pm 7,19$ & \\
\hline \multirow{2}{*}{ Smoking(packyear) } & Yes & 22 & $18,86 \pm 16,82$ & \multirow{2}{*}{${ }^{* *} 0,015$} \\
\hline & No & 53 & $11,60 \pm 8,42$ & \\
\hline \multirow{2}{*}{ Leukocyte $\left(\mathrm{mm}^{3}\right)$} & Yes & 21 & $7706 \pm 1407$ & \multirow{2}{*}{${ }^{*} 0,460$} \\
\hline & No & 50 & $8012 \pm 1648$ & \\
\hline \multirow{2}{*}{ Neutrophil (mL) } & Yes & 21 & $4727 \pm 1093$ & \multirow{2}{*}{ *0,934 } \\
\hline & No & 50 & $4703 \pm 1241$ & \\
\hline \multirow{2}{*}{ Lymphocyte (mL) } & Yes & 21 & $2081 \pm 545$ & \multirow{2}{*}{${ }^{* *} 0,022$} \\
\hline & No & 50 & $2444 \pm 599$ & \\
\hline \multirow{2}{*}{ Thrombocyte $\left(\mathrm{mm}^{3}\right)$} & Yes & 21 & $262300 \pm 50255$ & \multirow{2}{*}{${ }^{*} 0,462$} \\
\hline & No & 50 & $253800 \pm 40460$ & \\
\hline \multirow{2}{*}{ NLR } & Yes & 21 & $2,38 \pm 0,70$ & \multirow{2}{*}{${ }^{* *} 0,016$} \\
\hline & No & 50 & $1,97 \pm 0,59$ & \\
\hline \multirow{2}{*}{ TLR } & Yes & 21 & $133,79 \pm 39,06$ & \multirow{2}{*}{$* * * 0,005$} \\
\hline & No & 50 & $109,03 \pm 29,00$ & \\
\hline \multirow{2}{*}{ ESR $(\mathbf{m m})$} & Yes & 20 & $4,73 \pm 2,81$ & \multirow{2}{*}{$* * 0,024$} \\
\hline & No & 40 & $3,23 \pm 1,69$ & \\
\hline \multirow{2}{*}{ CRP (mg/L) } & Yes & 21 & $0,39 \pm 0,31$ & \multirow{2}{*}{${ }^{*} 0,089$} \\
\hline & No & 40 & $0,24 \pm 0,23$ & \\
\hline \multirow{2}{*}{ Albumin (g/dl) } & Yes & 20 & $4,42 \pm 0,41$ & \multirow{2}{*}{$* * * 0,008$} \\
\hline & No & 51 & $4,67 \pm 0,29$ & \\
\hline \multirow{2}{*}{ FEV1(L) } & Yes & 22 & $3,08 \pm 0,68$ & \multirow{2}{*}{${ }^{*} 0,154$} \\
\hline & No & 52 & $3,29 \pm 0,53$ & \\
\hline FEV1 (\%) & Yes & 22 & $84,5 \pm 16,5$ & $* 0054$ \\
\hline (1) & No & 52 & $91,2 \pm 11,9$ & 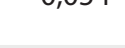 \\
\hline EVC (I) & Yes & 22 & $3,85 \pm 0,85$ & *0 121 \\
\hline rve(L) & No & 52 & $4,15 \pm 0,71$ & 0,121 \\
\hline FVC (\%) & Yes & 22 & $87,1 \pm 17,4$ & $* * 0044$ \\
\hline & No & 52 & $94,4 \pm 12,0$ & 0,044 \\
\hline FFV1/FVC & Yes & 22 & $80,6 \pm 8,3$ & *0 832 \\
\hline 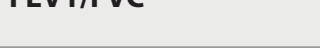 & No & 52 & $80,2 \pm 7,6$ & 0,032 \\
\hline Student $t$ test & & & ${ }^{*} \mathrm{NS}$ & \\
\hline NLR: Neutrophil Lymphocy & & & ${ }^{* *} p<0,05$ & \\
\hline TLR: Thrombocyte Lympho & & & ${ }^{* * *} p<0,01$ & \\
\hline $\mathrm{n}$ : number of patients & & & & \\
\hline SD: Standart Deviation & & & & \\
\hline Hilar LAP: Hilar Lymphaden & & & & \\
\hline
\end{tabular}

of inflammatory pathologies (such as pediatric appendicitis) and postoperative complications [12]. There are studies showing that there is a relationship between TLP and patients with chronic obstructive pulmonary disease, pulmonary embolism and tuberculosis $[14,15]$. In the study conducted by Karataş et al, it was found that NLR and TLR values were statistically significantly higher in pneumoconiosis patients with ILO categories 2 and 3 compared to the control group [5]. As a result of our study, we found a significant increase in NLR and TLR values in chronic pneumoconiosis patients with hilar lymphadenopathy. These findings indicate that the inflammation process is 
more active in pneumoconiosis patients with hilar lymphadenopathy than in those without.

Albumin is a natural plasma protein with a mean half-life of about 18 to 19 days, synthesized in healthy individuals only in liver tissue at a rate of 9 to $14 \mathrm{~g}$ per day [10]. The direct effect of albumin on mortality and morbidity is well known in the medical community. It is a negative acute phase reactant and has prognostic value. In a meta-analysis of 90 cohort studies, $1 \mathrm{~g} / \mathrm{dl}$ reduction in serum albumin resulted in 137\% increase in mortality, $89 \%$ increase in morbidity and $71 \%$ increase in hospital stay [11]. In our study, there was a statistically significant decrease in albumin values in pneumoconiosis patients with hilar lymphadenopathy. There was no pathology that would cause low albumin in these patients (such as chronic liver disease, chronic kidney disease, active infection).

In general, pulmonary function tests in pneumoconiosis show a typical restrictive pattern with a reduction in forced vital capacity (FVC) but maintaining the FEV1 / FVC ratio. There may be situations in which chronic airflow obstruction may accompany this situation [16]. Significant reduction in FVC (\%) of patients with hilar lymphadenopathy is a sign in favor of restriction. Of course, it should not be forgotten that the ILO categories of our patients with hilar lymphadenopathy are high.

Smoking plays a role in the etiology of many lung diseases. Among these diseases, there are chronic emphysema, lung cancers as well as desquamative interstitial pneumonia (DIP) and respiratory bronchiolitis-interstitial lung disease (RB-ILD) [17]. Crystalline silica is known to cause faster and more severe damage to the lungs in the presence of cigarette smoke. In our study, the significantly higher cigarette exposure in pneumoconiosis patients with hilar lymphadenopathy supports the damage caused by smoking.

Of course, our study had its drawbacks: only male patients (demographically), smoking (confounding factor), use of non-contrast computed tomography (in terms of imaging mediastinal pathologies).

In conclusion, we investigated the frequency of hilar lymphadenopathy and related factors in pneumoconiosis patients who came to our outpatientclinicinthepast5years.Inpneumoconiosis patients with hilar lymphadenopathy, a significant increase in NLR and PLR, a significant decrease in lymphocyte and albumin levels are evidence of continuing inflammation in the chronic process, and may be a sign of gradually decreasing immune response and accelerating the progression to pulmonary fibrosis. In this study, we tried to view hilar lymphadenopathy as a finding indicating an increase in parenchymal inflammation rather than the result of the pathophysiology of silicosis. We hope that more studies on this topic will be needed in the future.

\section{ACKNOWLEDGMENTS}

I thank my wife (Zeynep HASANLI) for supporting me in this process.

\section{CONFLICT Of INTEREST STATEMENT}

There is no conflict of interest regarding our study.
[1] Remy-Jardin M, Degreef JM, Beuscart R, et al. Coal worker's pneumoconiosis: CT assessment in exposed workers and correlation with radiographic findings. Radiology. 1990; 177(2): 363-71.

[2] Alıcı NŞ, Çimrin A, Beyan AC. Pneumoconiosis in different sectors and their differences in Turkey. Tuberk Toraks. 2016; 64(4): 275-282.

[3] Ergün D, Ergün R, Evcik E, et al. The relation between the extent of radiological findings and respiratory functions in pneumoconiosis cases of dental technicians who are working in Ankara. Tuberk Toraks. 2016; 64(2): 127-136.
[4] Gu HL, Fei WP, Hua PY, et al. Modified Glasgow Prognostic Score, and Neutrophil/lymphocyte and Platelet/ lymphocyte Ratios in Different Stages of Silicosis. Biomed Environ Sci. 2019; 32(5): 376-379.

[5] Karataş $M$, Gündüzöz $M$, Öziş $T N$, et al. Neutrophil to lymphocyte ratio and platelet to lymphocyte ratio as haematological indices of inflammatory response in ceramic workers' silicosis. Clin Respir J. 2019; 13: 159-165.

[6] Chong S, Lee KS, Chung MJ, et al. Pneumoconiosis: Comparison of Imaging and Pathologic Findings. RadioGraphics. 2006; 26: 59-77. 
[7] Nin CS, Souza VVS, Amaral RH, et al. Thoracic lymphadenopathy in benign diseases: A state of the art review. Respiratory Medicine. 2016; 112: 10-17.

[8] Cox-Ganser JM, Burchfiel CM, Fekedulegn D, et al. Silicosis in Lymph Nodes: The Canary in the Miner? Journal of Occupational and Environmental Medicine. 2009; 51(2): 164-169.

[9] Zhang R, Wu X, Hu W, et al. Neutrophil-to-lymphocyte ratio predicts hemorrhagic transformation in ischemic stroke: $\mathrm{A}$ meta-analysis. Brain Behav. 2019; 9(9): e01382.

[10] Vincent JL, Russell JA, Jacob $M$, et al. Albumin administration in the acutely ill: what is new and where next? Crit Care. 2014; 18(4): 231.

[11] Vincent JL, Dubois MJ, Navickis RJ, et al. Hypoalbuminemia in acute illness: is there a rationale for intervention? A meta-analysis of cohort studies and controlled trials. Ann Surg. 2003; 237:319-334.

[12] Forget $P$, Khalifa C, Defour JP, et al. What is the normal value of the neutrophil-to-lymphocyte ratio? BMC Res Notes. 2017; 10(1): 12.

[13] Murray J, Webster I, Reid G, et al. The relation between fibrosis of hilar lymph glands and the development of parenchymal silicosis. Br J Ind Med. 1991; 48: 267-269.
[14] Kurtipek E, Bekci TT, Kesli R, et al. The role of neutrophillymphocyte ratio and platelet-lymphocyte ratio in exacerbation of chronic obstructive pulmonary disease. J Pak Med Assoc. 2015; 65: 1283-1287.

[15] Karataş MB, Ijpek G, Onuk T, et al. Assessment of prognostic value of neutrophil to lymphocyte ratio and platelet to lymphocyte ratio in patients with pulmonary embolism. Acta Cardiol Sin. 2016; 32: 313.

[16] DeLight N, Sachs H. Pneumoconiosis. Treasure Island (FL): StatPearls Publishing; 2021.

[17] Rivera-Ortega $P$, Molina-Molina $M$. Interstitial Lung Diseases in Developing Countries. Ann Glob Health. 2019; 85(1): 4.

[18] Satija B, Kumar S, Ojha UC and Gothi D. Spectrum of high-resolution computed tomography imaging in occupational lung disease. Indian J Radiol Imaging. 2013; 23(4): 287-296.

[19] Nin CS, Silveira de Souza VV, Amaral RH, et al. Thoracic lymphadenopathy in benign diseases: A state of the art review. Respiratory Medicine. 2016; 112: 10-17. 